\title{
CHARACTERIZATION OF $L^{2}(\mathscr{M})$ \\ FOR INJECTIVE W*-ALGEBRAS $\mathscr{M}$
}

\author{
LOTHAR M. SCHMITT
}

\begin{abstract}
.
We characterize matrix ordered standard forms $\left(\mathscr{M}, \mathscr{H}, \mathscr{H}_{n}^{+}, n \in \mathrm{N}\right)$ of $\mathrm{W}^{*}$-algebras $\mathscr{M}$ by the following property: every matrix ordered subspace of $\left(\mathscr{H}, \mathscr{H}_{n}^{+}\right)$generated by the face of a single element in $\mathscr{H}_{1}^{+}$must be completely isomorphic to some $\mathrm{W}^{*}$-algebra.

We relate the completely positive extension property (injectivity) of matrix ordered spaces, which are dual spaces, to a matricial analogue of the Riesz interpolation property introduced by Wittstock.

As consequences we obtain: if $\left(\mathscr{H}, \mathscr{H}_{n}^{+}, n \in \mathrm{N}\right)$ is a Hilbert space, which is matrix ordered by a family of selfdual cones $\mathscr{H}_{n}^{+} \subset \mathscr{H} \otimes M_{n}$, then $\left(\mathscr{H}, \mathscr{H}_{n}^{+}\right)$is (finitely) injective or has the matricial Riesz interpolation property if and only if there exists an injective $\mathrm{W}^{*}$-algebra $\mathscr{M}$, such that $\left(\mathscr{M}, \mathscr{H}, \mathscr{H}_{n}^{+}, n \in \mathrm{N}\right)$ is a matrix ordered standard form.
\end{abstract}

\section{Introduction.}

Function spaces like $C(X)$ or $L^{p}(X, \mu)$ - classical objects of functional analysis - carry a natural order structure. A unifying treatment of these order structures is the theory of Banach lattices. In this setting the spaces mentioned above have been given abstract characterizations [19]. For example consider a Hilbert lattice $\mathscr{H}$. $\mathscr{H}$ is isomorphic to $L^{2}(X, \mu)$, where $X$ is a locally compact space and $\mu$ is a strictly positive Radon measure on $X-$ Schaefer [19]. $\mathscr{H}$ has a selfdual cone $\mathscr{H}^{+}=L^{2}(X, \mu)^{+}$. Every face in $\mathscr{H}^{+}$ generated by a single function $f \geqq 0$ is isomorphic to some $L^{\infty}\left(X_{1}, \mu_{1}\right)^{+}$. Conversely if $\mathscr{H}$ is a Hilbert space with a selfdual cone $\mathscr{H}^{+}$and every singleton face of $\mathscr{H}^{+}$is isomorphic to some $L^{\infty}\left(X_{1}, \mu_{1}\right)^{+}$, then it is a Hilbert lattice. In the first part of this paper we shall prove a similar statement for non-commutative $L^{2}$-spaces. These are the Hilbert spaces arising in standard forms of $\mathrm{W}^{*}$-algebras introduced by Araki [2], Connes [8] and Haagerup [13]. Choi and Effros [6] introduced matrix ordered spaces as an appropriate means to handle non-commutative order. The Hilbert space in a standard form carries a natural matrix order. In [21] Wittstock and the

Received February 7, 1984; in revised form May 30, 1984. 
author characterized those matrix ordered Hilbert spaces $\mathscr{H}$ with selfdual cones $\mathscr{H}_{n}^{+} \subset \mathscr{H} \otimes M_{n}$ arising from standard forms of $\mathrm{W}^{*}$-algebras by a projection property: for every closed face in $\mathscr{H}_{n}^{+}$there exists a projection from $\mathscr{H}_{n}^{+}$onto this face. We relate this property to the following: every matrix ordered subspace of $\left(\mathscr{H}, \mathscr{H}_{n}^{+}\right)$generated by the face of a single element in $\mathscr{H}_{1}^{+}$must be completely isomorphic to some $\mathrm{W}^{*}$-algebra.

As well as order structures and integration theory are related in the commutative and the non-commutative situation, there is a fundamental link between order structures and extension properties. An injective Banach space is isometrically isomorphic to $C(X)$, the bounded continuous functions on a Stonean space $X$. Goodner, Kelley and Nachbin (see [4], [16]) proved this in the real and Hasumi [14] in the complex case. A result of Choi and Effros [6] says, that an injective matrix ordered space $R$ with archimedean cones $M_{n}(R)^{+}$and an order unit is completely isomorphic to an injective $C^{*}$-algebra. If $R$ is a dual Banach space, then it is completely isomorphic to an injective $W^{*}$-algebra. Choi and Effros [6], [11] also proved, that for a $\mathrm{W}^{*}$-algebra $R$ injectivity and the following extension property are equivalent: every completely positive map $\varphi: N_{2 n} \rightarrow R$ has a completely positive extension to $M_{2 n}$. Wittstock [26] has shown, that for $\mathrm{W}^{*}$-algebras injectivity, the matricial Hahn-Banach property and a matricial analogue of the Riesz interpolation property are equivalent. In the second part of this paper we study Arveson spaces, i.e. matrix ordered spaces, which have the completely positive extension property in the category of matrix ordered spaces. We shall prove, that for a matrix ordered space $R$, which is the dual space of a matrix ordered, $\lambda$-generating Banach space, all the properties mentioned above are equivalent to $R$ being an Arveson space.

The results of part one and part two yield to characterizations of matrix ordered standard forms [21] of injective $\mathrm{W}^{*}$-algebras, which we shall obtain in part three.

\section{Notation.}

Suppose that $V$ is a complex vector space with an involution *. We shall write $V_{h}$ for the real subspace of selfadjoint elements $v=v^{*}$. Then $V=V_{h} \oplus i V_{h}$. Let $M_{m, n}$ be the space of complex $m \times n$ matrices and $M_{n}$ be the $n \times n$ matrices; $n, m \in N$. We shall write $1_{n}$ for the unit element in $M_{n}$. $M_{m, n}$ always carries its natural involution st: $\alpha \rightarrow \alpha^{*}$. If

$$
V_{m, n}=M_{m, n}(V)=V \otimes M_{m, n}
$$

is the space of $m \times n$ matrices with entries in $V$, then we define the involution 


$$
(\cdot)^{*}: V_{m, n} \rightarrow V_{n, m} \text { as }\left[v_{\mu v}\right]^{*}=\left[v_{v \mu}^{*}\right]
$$

If we write

$$
V_{n}=M_{n}(V)=V \otimes M_{n}=V_{n, n},
$$

then

$$
\left(V_{n}\right)_{h}=V_{h} \otimes_{\mathrm{R}}\left(M_{n}\right)_{h} .
$$

Suppose $v \in V_{n}$ and $w \in V_{m}$. We define:

$$
v \oplus w=\left(\begin{array}{cc}
v & 0 \\
0 & w
\end{array}\right) \in V_{n+m} .
$$

Especially we define $\sigma_{n, n}=1_{n} \oplus\left(-1_{n}\right) \in M_{2 n}$. For $v \in V_{n}$ and $w \in W_{n}$ we let

$$
v \times w=\sum_{\mu \nu} v_{\mu \nu} \otimes w_{\mu \nu} \in V \otimes W
$$

In particular

$$
v \times \alpha=\sum_{\mu \nu} \alpha_{\mu v} v_{\mu v} \text { for } v \in V_{n}, \alpha \in M_{n}
$$

Now let

$$
N_{2 n}:=\left\{y \in M_{2 n} \mid y \times \sigma_{n, n}=0\right\} .
$$

$N_{2 n}$ is a matrix system in $M_{2 n}$ [6].

A complex linear map $\varphi: V \rightarrow W$ is called selfadjoint if $\varphi\left(v^{*}\right)=\varphi(v)^{*}$. Put

$$
\varphi_{n}=\varphi \otimes \mathrm{id}_{M_{n}}: V_{n} \rightarrow W_{n},\left[v_{\mu \nu}\right] \rightarrow\left[\varphi\left(v_{\mu \nu}\right)\right] .
$$

A complex vectorspace $V$ with an involution * is said to be matrix ordered, if each $\left(V_{n}\right)_{h}$ is partially ordered by a cone $V_{n}^{+}$and the following transformation law yields: if $\alpha \in M_{m, n}$ then $\alpha V_{n}^{+} \alpha^{*} \subset V_{m}^{+}$. If $V$ and $W$ are matrix ordered and $\varphi: V \rightarrow W$ is a selfadjoint map, then we say that $\varphi$ is completely positive if $\varphi_{n}\left(V_{n}^{+}\right) \subset W_{n}^{+}$for every $n \in N$. Every completely positive map $\varphi: M_{n} \rightarrow M_{m}$ can be written as

$$
\begin{gathered}
\varphi(\beta)=\alpha \times \beta=\beta \times \alpha^{\prime}=\Sigma \gamma_{i} \beta \gamma_{i}^{*} ; \\
\alpha, \alpha^{\prime} \in M_{m \cdot n}^{+}, \gamma_{i} \in M_{n, m}, \beta \in M_{n} .
\end{gathered}
$$

Conversely the last three expressions always define completely positive maps $M_{n} \rightarrow M_{m}$. This follows from [5, Theorem 1] and [6, Lemma 4.1]. As usual we shall suppress the indices $n=m=k=, \ldots,=1$, if there is no possibility of misunderstanding. 


\section{Matrix ordered standard forms of $\mathbf{W}^{*}$-algebras.}

Let $\mathscr{M}$ be a $\mathrm{W}^{*}$-algebra and $\varphi$ a normal, faithful, semifinite weight on $\mathscr{M}$. Using the well known results from [23], [22], [8], [13] we obtain the achieved left Hilbert algebra $\mathscr{A}_{\varphi}$, the modular operator $\Delta_{\varphi}$, the canonical involution $J$ and the standard form $\left(\mathscr{M}, \mathscr{H}, J, \mathscr{H}^{+}\right)$, which is unique up to spatial isomorphisms. If tr denotes the trace on $M_{n}$, then $\mathscr{A}_{\varphi} \otimes M_{n}$ is the achieved left Hilbert algebra corresponding to the weight $\varphi \otimes \operatorname{tr}$ on $\mathscr{M}_{n}$. It generates the standard form $\left(\mathscr{M}_{n}, \mathscr{H}_{n}, J_{n}, \mathscr{H}_{n}^{+}\right)$, where $\mathscr{M}_{n}$ operates on $\mathscr{H}_{n}$ by matrix multiplication from the left. Lemma 1.1. of [21] shows that

$$
a J_{n, m} a J_{m} \mathscr{H}_{m}^{+} \subset \mathscr{H}_{n}^{+}
$$

for every $a \in \mathscr{M}_{n, m} ; n, m \in N$. In particular $\mathscr{H}$ is matrix ordered by the cones $\mathscr{H}_{n}^{+}$, which implies that $J_{n}=J \otimes$ st [21, Lemma 1.3]. Consequently we call $\left(\mathscr{M}, \mathscr{H}, \mathscr{H}_{n}^{+}, n \in \mathrm{N}\right)$ a matrix ordered standard form [21, Definition 1.4].

Now suppose that $\mathscr{H}$ is some Hilbert space, which is matrix ordered by a family of selfdual cones $\mathscr{H}_{n}^{+} \subset \mathscr{H}_{n}$. Consider a face $F \subset \mathscr{H}_{n}^{+}$for some $n \in \mathrm{N}$, i.e. a hereditary subcone of $\mathscr{H}_{n}^{+}$. If one defines the orthogonal face as

$$
F^{\perp}=\left\{\xi \in \mathscr{H}_{n}^{+} \mid\langle\xi, F\rangle=0\right\},
$$

then $F$ is said to be completed if $F=F^{\perp \perp}$. Writing $P_{F}$ for the selfadjoint projection on the closed subspace of $\mathscr{H}$ generated by $F$, we call $F$ a projectable face if $P_{F} \mathscr{H}_{n}^{+}=F$. If $\xi \in \mathscr{H}_{n}^{+}$, then

$$
F_{\xi}=\left\{\eta \in \mathscr{H}_{n}^{+} \mid \exists \lambda \in \mathrm{R}^{+}, 0 \leqq \eta \leqq \lambda \xi\right\}
$$

is the face generated by $\xi$. It is easy to see, that for $\xi_{m}=\xi \otimes 1_{m} \in \mathscr{H}_{n \cdot m}^{+}$

$$
\operatorname{span}_{\mathrm{C}} F_{\xi_{m}}=\left(\operatorname{span}_{\mathrm{C}} F_{\xi}\right)_{m}
$$

and $\left(\operatorname{span}_{C} F_{\xi}, F_{\xi_{m}}\right)$ is a matrix ordered linear space.

In [21] we proved the following Theorem which characterizes those matrix ordered Hilbert spaces, which arise from matrix ordered standard forms of $\mathrm{W}^{*}$-algebras:

Theorem 1.1. Suppose that $\mathscr{H}$ is a matrix ordered Hilbert space with selfdual cones $\mathscr{H}_{n}^{+} \subset \mathscr{H}_{n}$. There exists a $\mathrm{W}^{*}$-algebra $\mathscr{M}$ on $\mathscr{H}$ such that $\left(\mathscr{M}, \mathscr{H}, \mathscr{H}_{n}^{+}, n \in \mathrm{N}\right)$ is a matrix ordered standard form, if and only if for every $n \in \mathrm{N}$ every completed face $\mathrm{F} \subset \mathscr{H}_{n}^{+}$is projectable.

In fact $\mathscr{M}$ is the matrix multiplier algebra of $\left(\mathscr{H}, \mathscr{H}_{n}^{+}\right)$[21, Definition 2.1 and Theorem 4.3]. If one is interested in a further exploration of the facial structure of $\left(\mathscr{H}, \mathscr{H}_{n}^{+}\right)$and looks at [8] or [22, section 10.24 , Lemma 3$]$ the following Definition is at hand: 
Definition 1.2. A Hilbert space $\mathscr{H}$, which is matrix ordered by selfdual cones $\mathscr{H}_{n}^{+} \subset \mathscr{H}_{n}$, has property $\mathscr{F}$, if for every $\xi \in \mathscr{H}_{1}^{+}$the linear, matrix ordered space $\left(\operatorname{span}_{\mathrm{C}} F_{\xi}, F_{\xi_{n}}, n \in \mathrm{N}\right)$ is completely order isomorphic to some $\mathrm{W}^{*}$-algebra $\mathscr{M}_{\xi}$. To be precise we have a linear, invertible map $\Phi: \mathscr{M}_{\xi} \rightarrow \operatorname{span}_{\mathrm{C}} F_{\xi}$, which satisfies $\Phi_{n}\left(\left(\mathscr{M}_{\xi}\right)_{n}^{+}\right)=F_{\xi_{n}}$ and $\Phi(1)=\xi$.

Let $\eta \in \mathscr{H}_{n}^{+}$. Then we have a $\gamma \in \mathscr{H}_{1}^{+}$such that $0 \leqq \eta \leqq \gamma \otimes 1_{n}$. If $\left(\mathscr{H}, \mathscr{H}_{n}^{+}\right)$has property $\mathscr{F}$, then $\left(\operatorname{span}_{\mathrm{C}} F_{\gamma}, F_{\gamma_{n}}\right)$ is completely isomorphic to a $\mathrm{W}^{*}$-algebra $\mathscr{M}_{\gamma}$. Since the linear, matrix ordered space generated by the face of an element in $\left(\mathscr{M}_{\gamma}\right)_{n}^{+}$is completely isomorphic to some other $\mathrm{W}^{*}$ algebra we obtain a linear, completely positive isomorphism

$$
\Phi:\left(\mathscr{M}_{\eta},\left(\mathscr{M}_{\eta}\right)_{n}^{+}\right) \rightarrow\left(\operatorname{span}_{\mathrm{C}} F_{\eta}, F_{\eta_{n}}\right) .
$$

Using that every Hilbert space $\mathscr{H}$ with selfdual cone $\mathscr{H}^{+}$is order complete $[18$, Chapter $\mathrm{V}$, section 4.3$]$ it is easy to see that such a map $\Phi$ occurring above is $\sigma\left(\mathscr{M}_{\xi}, \mathscr{M}_{\xi *}\right)-\sigma(\mathscr{H}, \mathscr{H})$ continuous. The main result of this section is:

THEOREM 1.3. Suppose that $\mathscr{H}$ is a matrix ordered Hilbert space with selfdual cones $\mathscr{H}_{n}^{+} \subset \mathscr{H}_{n}, n \in \mathrm{N}$. Let $\mathscr{M}$ be the matrix multiplier algebra of $\left(\mathscr{H}, \mathscr{H}_{n}^{+}\right)$. Then the following three statements are equivalent:

(a) $\left(\mathscr{M}, \mathscr{H}, \mathscr{H}_{n}^{+}\right)$is a matrix ordered standard form.

(b) Every completed face $F \subset \mathscr{H}_{n}^{+}$is selfdual in $P_{F} \mathscr{H}, n \in \mathrm{N}$.

(c) $\left(\mathscr{H}, \mathscr{H}_{n}^{+}\right)$has property $\mathscr{F}$.

We need some Lemmas for the proof of Theorem 1.3:

LEMMA 1.4. Suppose that $\left(\mathscr{M}, \mathscr{H}, \mathscr{H}_{n}^{+}, n \in \mathrm{N}\right)$ is a matrix ordered standard form of a $\mathrm{W}^{*}$-algebra and $\xi \in \mathscr{H}_{1}^{+}$.

(a) If $\mathscr{M}$ is $\sigma$-finite and $\xi$ is cyclic and separating for $\mathscr{M}$, then the map

$$
\varphi: x \rightarrow \Delta_{\omega_{\xi}}^{1 / 4} x \xi,
$$

is a completely positive isomorphism of $\left(\mathscr{M}, \mathscr{M}_{n}^{+}\right)$onto $\left(\operatorname{span} F_{\xi}, F_{\xi_{n}}\right)$ satisfying $\Phi_{n}\left(\mathscr{M}_{n}^{+}\right)=F_{\xi_{n}}$ and $\Phi(1)=\xi$.

(b) If $\mathscr{M}$ is arbitrary and $p=\operatorname{supp}\left(\omega_{\xi}\right)$ then there exists a completely positive isomorphism $\Phi$ of $\left(p \mathscr{M} p,(p \mathscr{M} p)_{n}^{+}\right)$onto $\left(\operatorname{span}_{\mathrm{C}} F_{\xi}, F_{\xi_{n}}\right)$ satisfying $\Phi_{n}\left((p \mathscr{M} p)_{n}^{+}\right)=F_{\xi_{n}}$ and $\Phi(p)=\xi$.

Proof. (a) $\xi_{n}=\xi \otimes 1_{n}$ is cyclic and separating for $\mathscr{M}_{n}$ and

$$
\Delta_{\omega_{\xi_{n}}}^{1 / 4}=\Delta_{\omega_{\xi}}^{1 / 4} \otimes 1_{n}
$$


by [23, Theorem 11.1]. So (a) follows from [22, section 10.24, Lemma 3].

In order to prove (b), let $q=p J p J$. Then by [13, Lemma 2.6] ( $q \mathscr{M} q$, $\left.q \mathscr{H}, q_{n} \mathscr{H}_{n}^{+}, n \in \mathrm{N}\right)$ is a matrix ordered standard form and $\xi \in q \mathscr{H}$ is cyclic and separating for $q \mathscr{M} q$. It follows from the proof of [21, Proposition 3.4], that $F_{\xi} \subset q \mathscr{H}^{+}$. Now we can apply (a) to obtain a completely positive isomorphism between $q \mathscr{M} q$ and $\operatorname{span}_{\mathrm{C}} F_{\xi}$. Observing that $q \mathscr{M} q$ and $p \mathscr{M} p$ are completely isomorphic, we are done.

If $\mathscr{H}^{+}$is a selfdual cone in a Hilbert space $\mathscr{H}$, then $\mathscr{H}^{+}$uniquely determines an involution $J$ on $\mathscr{H}$. It is well known [8], [15], that for $\xi \in \mathscr{H}^{J}=\mathscr{H}_{h}$ there exist unique $\xi^{+}, \xi^{-} \in \mathscr{H}^{+}$satisfying $\xi=\xi^{+}-\xi^{-}$, $\xi^{+} \perp \xi^{-}$. We call this the Jordan decomposition of $\xi$. In addition we define $|\xi|=\xi^{+}+\xi^{-}$.

Lemma 1.5. Suppose $\mathscr{H}^{+}$is a selfdual cone in a Hilbert space $\mathscr{H}$ and $F \subset \mathscr{H}^{+}$is a face in $\mathscr{H}^{+}$. Then $P_{F} \mathscr{H}^{\mathrm{J}} \subset \mathscr{H}^{\mathrm{J}}$ and the following three conditions are equivalent:

(a) $F$ is a projectable face in $\mathscr{H}^{+}$.

(b) If $\xi \in\left(\operatorname{span}_{R} F\right)^{-}$, then there exist $\xi^{+}, \xi^{-} \in F$ such that $\xi=\xi^{+}-\xi^{-}$and $\xi^{+} \perp \xi^{-}$.

(c) $F$ is selfdual in $P_{F} \mathscr{H}$.

Since this Lemma is more or less known [15], we omit the proof as well as the proof of the next Lemma.

Lemma 1.6. Suppose $\mathscr{H}^{+}$is a selfdual cone in a Hilbert space $\mathscr{H}$ and $\xi \in \mathscr{H}^{J}$. Then $|\xi|$ is a minimal element of $\{\eta \mid \pm \xi \leqq \eta\}$, which has minimal norm in this set.

LemMa 1.7. Suppose $\mathscr{H}$ is a matrix ordered Hilbert space with selfdual cones $\mathscr{H}_{n}^{+}, n \in \mathrm{N}$, which has property $\mathscr{F}$.

(a) If $G$ is a closed face in $\mathscr{H}_{n}^{+}$and $\xi \in \operatorname{span}_{\mathrm{R}} G$, then $|\xi| \in G$.

(b) If $\xi=\xi^{+}-\xi^{-}$is the Jordan decomposition of $\xi \in \operatorname{span}_{R} G$, then $\xi^{ \pm} \in G$.

(c) $\operatorname{span}_{\mathrm{R}} G=\left(\operatorname{span}_{\mathrm{R}} G\right)^{-}$.

Proof. Let $\xi=\xi_{1}-\xi_{2}, \xi_{1,2} \in G$. Then $\pm \xi \leqq \xi_{1}+\xi_{2}=: \eta \in G$. By hypothesis there exists a $W^{*}$-algebra $\mathscr{M}_{|\xi|+\eta}$ and an order isomorphism

$$
\Phi: \mathscr{M}_{|\xi|+\eta} \rightarrow \operatorname{span}_{\mathrm{C}} F_{|\xi|+\eta}
$$

satisfying $\Phi(1)=|\xi|+\eta$. Let $z=\Phi^{-1}(|\xi|), y=\Phi^{-1}(\eta)$ and $x=\Phi^{-1}(\xi)$. If $p=\operatorname{supp} y$ is the support projection of $y$, then $z$ and $p$ commute and we have: 


$$
\begin{aligned}
& 0 \leqq \pm x+y \leqq 2 y \leqq 2\|y\| p \\
\Rightarrow & ( \pm x+y) p^{\perp}=0 \\
\Rightarrow & \pm x= \pm p x p \leqq p z p=z p \leqq z .
\end{aligned}
$$

Now Lemma 1.6 implies that $z p=z$. Denote the spectral resolution of $y$ by $E$ and put $p_{n}=E([1 / n, 1]), n \in \mathrm{N}$. Then $p_{n} \uparrow p$ and $p_{n} \leqq n y$. The continuity of $\Phi$ implies that $\Phi(p) \in G$ and $\Phi(p) \geqq|\xi| \in G$. This gives (a) and (b). Suppose $\xi_{n} \in \operatorname{span}_{\mathrm{R}} G, n \in \mathrm{N}$ such that

$$
\sum_{n=1}^{\infty}\left\|\xi_{n}\right\|=\sum_{n=1}^{\infty}\left\|\left|\xi_{n}\right|\right\|<\infty
$$

Then

$$
\sum_{n=1}^{\infty} \xi_{n}^{ \pm} \leqq \sum_{n=1}^{\infty}\left|\xi_{n}\right| \in G \text { and } \sum_{n=1}^{\infty} \xi_{n} \in \operatorname{span}_{\mathrm{R}} G .
$$

This proves (c).

Proof of Theorem 1.3: (a) $\Leftrightarrow(\mathrm{b})$, is Theorem 1.1 and Lemma 1.5.

(a) $\Rightarrow$ (c) is Lemma 1.4 and

(c) $\Rightarrow$ (a) is Theorem 1.1, Lemma 1.7 and Lemma 1.5 .

\section{Arveson spaces.}

The class of injective $\mathrm{W}^{*}$-algebras proved to be a very nice subclass of all $\mathrm{W}^{*}$-algebras, which possesses remarkable stability properties [10]. They arise in theoretical physics and as biduals of nuclear $\mathrm{C}^{*}$-algebras [7]. Consequently they have been an object of intensive study and have been given many characterizations [3], [6], [9], [11], [26]. In this section, which is closely related to [6] and [26], we consider the completely positive extension property in the category of matrix ordered spaces. We obtain several characterizations of this property for those spaces, we are interested in.

In what follows $R$ is always supposed to be a matrix ordered space. A subspace $S \subset R$ is called cofinal, if $S=S^{*}$ and for every $x \in R_{h}$ there exists $y \in S_{h}$, such that $x \leqq y$.

Definition 2.1. We call $R$ an Arveson space, if for every matrix ordered space $Q$ and every cofinal subspace $S \subset Q$ every completely positive, linear map $\varphi: S \rightarrow R$ can be extended to a completely positive, linear map $\Phi: Q \rightarrow R$.

A cofinal subspace $S \subset R$ is an Arveson space, if and only if there exists a completely positive projection $P: R \rightarrow S$. 
Definition 2.2. Suppose $x \in\left(R_{2 n}\right)_{h} ; n \in N$. $x$ is called $\sigma_{n, n}$-positive, if for every $k$-tupel $\left(\gamma_{1}, \ldots \gamma_{k}\right) \in M_{2 n, m}^{k}, k, m \in N$ satisfying

$$
\sum_{\kappa=1}^{k} \gamma_{\kappa}^{*} \sigma_{n, n} \gamma_{\kappa}=0
$$

we have that

$$
\sum_{\kappa=1}^{k} \gamma_{\kappa}^{*} x \gamma_{\kappa} \geqq 0
$$

$r \in R_{h}$ is called a lower $\sigma_{n, n}$-bound of $x$, if $r \otimes \sigma_{n, n} \leqq x . R$ has the matricial Riesz interpolation property, if every $\sigma_{n, n}$-positive $x \in\left(R_{2 n}\right)_{h}$ has a lower $\sigma_{n, n}$-bound.

The matricial Riesz interpolation property first appeared in [26], where it is shown, that it characterizes injective $W^{*}$-algebras among matrix ordered order unit spaces, which are dual spaces. In [27] it is proved, that for uniformly complete ordered vector spaces the ordinary Riesz interpolation property implies its matricial analogue. A similar condition, that characterizes injectivity, is given in [6, Theorem 3.4].

Definition 2.3. Suppose that $R_{*}$ is a matrix ordered topological vector space - product topology on $M_{n}\left(R_{*}\right)$. Define

$$
\begin{aligned}
K_{2 n}\left(R_{*}\right)= & \left(R_{*} \otimes N_{2 n}\right) \cap M_{2 n}\left(R_{*}\right)^{+} \\
= & \left\{y \in M_{2 n}\left(R_{*}\right)^{+} \mid \sigma_{n, n} \times y=0\right\}, \\
L_{2 n}\left(R_{*}\right)= & \left(R_{*} \otimes N_{2 n}\right)^{+} \\
= & \left\{y \in M_{2 n}\left(R_{*}\right)^{+} \mid \text {ex. } \gamma \in\left(M_{2 n} \otimes M_{m}\right)^{+},\right. \\
& \mathscr{Y} \in M_{m}\left(R_{*}\right)^{+} \text {such that } \\
& \left.\sigma_{n, n} \times \gamma=0, \gamma \times \mathscr{Y}=y\right\} .
\end{aligned}
$$

$\boldsymbol{R}_{*}$ has the approximative factorisation property, if

$$
K_{2 n}\left(R_{*}\right) \subset L_{2 n}\left(R_{*}\right)^{-} \text {for every } n \in \mathrm{N} \text {. }
$$

The approximate factorisation property appears hidden in the proof of [6, Theorem 5.2]. Suppose that $R_{*}$ and $R$ are dual matrix ordered spaces in the sense of [6, page 175]. Using [6, Lemma 4.3] one gets:

$$
K_{2 n}\left(R_{*}\right) \simeq \mathrm{CP}_{\sigma}\left(R, N_{2 n}\right) \simeq \mathrm{CP}\left(N_{2 n}^{d}, R_{*}\right)
$$

where $\sigma$ means $\sigma\left(R, R_{*}\right)$-continuous.

By $[6$, Corollary 4.8$]$ we obtain: 
$R_{*}$ has the approximative factorisation property.

$\Leftrightarrow$ Every $\sigma\left(R, R_{*}\right)$-continuous, completely positive map from $R$ to $N_{2 n}$ has an approximative factorisation through $M_{m}$.

$\Leftrightarrow$ Every completely positive, linear map from $N_{2 n}^{d}$ to $R_{*}$ has an approximative factorisation through $M_{m}$.

Theorem 2.4. Suppose $R$ is a Banach space, which is the dual space of a matrix ordered Banach space $R_{*}$ with continuous involution. Suppose in addition, that $R_{*}$ is $\lambda$-generating for some $\lambda \geqq 1$, that is for $x \in\left(R_{*}\right)_{h}$, there exist $x_{1}, x_{2} \in R_{*}^{+}$such that $x=x_{1}-x_{2}$ and $\left\|x_{1}\right\|+\left\|x_{2}\right\| \leqq \lambda\|x\|$.

The following conditions are equivalent:

(I) $R$ is an Arveson space.

(II) $R$ is finitely injective, [6, page 165].

(III) Any completely positive, linear map $\varphi: N_{2 n} \rightarrow R$ has a completely positive, linear extension to $M_{2 n}$.

(IV) $R_{*}$ has the approximative factorisation property.

(V) $R$ has the matricial Riesz interpolation property.

(VI) $R$ has the matricial Hahn-Banach property, [25, page 135].

We need a Lemma:

LEMMA 2.5. (a) The dual cone of $L_{2 n}\left(R_{*}\right)$ in $\left(R_{2 n}\right)_{h}$ is the set of all $\sigma_{n, n^{-}}$ positive elements in $R_{2 n}$.

(b) If $y \in\left(R_{2 n}\right)_{h}$ is $\sigma_{n, n}$-positive, then $\varphi: N_{2 n} \rightarrow R, \alpha \mapsto \alpha \times y$ defines $a$ completely positive map.

Proof. a) If $y \in\left(R_{2 n}\right)_{h}$ is in the dual cone of $L_{2 n}\left(R_{*}\right)$, then $y \times \gamma \geqq 0$ for every $\gamma \in\left(M_{2 n} \otimes M_{n}\right)^{+}$with $\sigma_{n, n} \times \gamma=0$. Hence by [5, Theorem 1] and [6, Lemma 4.1], this is equivalent to:

$$
\begin{gathered}
\text { if } \gamma_{1}, \ldots \gamma_{k} \in M_{2 n, m} \text { such that } \sum_{\kappa=1}^{k} \gamma_{\kappa}^{*} \sigma_{n, n} \gamma_{\kappa}=0, \\
\text { then } \sum_{\kappa=1}^{k} \gamma_{\kappa}^{*} y \gamma_{\kappa} \geqq 0 .
\end{gathered}
$$

b) Suppose $\alpha \in M_{m}\left(N_{2 n}\right)^{+} \subset\left(M_{m} \otimes M_{2 n}\right)^{+}$. Then there exist finitely many $\gamma_{\kappa} \in M_{2 n, m}$ such that

$$
\begin{aligned}
& \alpha \times \beta=\sum_{\kappa} \gamma_{\kappa} \beta \gamma_{\kappa} \text { for every } \beta \in M_{2 n} . \\
& \alpha \times \sigma_{n, n}=\sum_{\kappa} \gamma_{\kappa} \sigma_{n, n} \gamma_{\kappa}=0 .
\end{aligned}
$$


Hence

$$
\alpha \times y=\sum_{\kappa} \gamma_{\kappa} y \gamma_{\kappa} \geqq 0
$$

Proof of Theorem 2.4. (III) $\Rightarrow$ (IV) is contained in the proof of $[6$, Theorem 5.2].

$(\mathrm{IV}) \Rightarrow(\mathrm{V})$. Suppose $x=\left[x_{\kappa \lambda}\right] \in M_{2 n}(R)_{h}$ is $\sigma_{n, n}$-positive. We define $\tau:\left(R_{*}\right)_{h} \rightarrow \mathrm{R}$ by

$$
\tau(y)=\inf \left\{\langle x, u\rangle \mid u \in M_{2 n}\left(R_{*}\right)^{+}, \sigma_{n, n} \times u=y\right\} .
$$

If (IV) holds, then

$$
\begin{aligned}
\tau(0) & =\inf \left\{\langle x, u\rangle \mid u \in K_{2 n}\left(R_{*}\right)\right\} \\
& =\inf \left\{\langle x, u\rangle \mid u \in L_{2 n}\left(R_{*}\right)\right\} \geqq 0 .
\end{aligned}
$$

Thus $\tau$ is a welldefined sublinear functional on $\left(R_{*}\right)_{h}$, which satisfies

$$
\begin{aligned}
\tau(y) & \leqq\left\langle x_{11}, y^{+}\right\rangle+\left\langle x_{2 n, 2 n}, y^{-}\right\rangle \\
& \leqq\left(\left\|x_{11}\right\|+\left\|x_{2 n, 2 n}\right\|\right) \lambda\|y\|,
\end{aligned}
$$

$y=y^{+}-y^{-}, y^{ \pm} \in R_{*}^{+}$suitably chosen. By the Hahn-Banach Theorem we have a $z \in R_{h}$ such that $\langle z, y\rangle \leqq \tau(y)$. If $y=\left[y_{\kappa \lambda}\right] \in M_{2 n}\left(R_{*}\right)^{+}$, then

$$
\left\langle z \otimes \sigma_{n, n}, y\right\rangle=\left\langle z, \sigma_{n, n} \times y\right\rangle \leqq \tau\left(\sigma_{n, n} \times y\right) \leqq\langle x, y\rangle .
$$

Hence $x$ is lower $\sigma_{n, n}$-bounded.

$(\mathrm{V}) \Leftrightarrow(\mathrm{VI})$ is [26, Satz 3.5].

(VI) $\Rightarrow$ (I) follows similarly as the proof of [26, Beispiel 2.3.3].

The equivalence $(\mathrm{I}) \Leftrightarrow(\mathrm{VI})$ holds for every matrix ordered space $R$ [20] and is a matricial analogue of the equivalence between the Krein-Rutmann extension Theorem for positive linear forms and the scalar Hahn-Banach Theorem. In the case, where $R$ is a W*-algebra, and in the case, where $R_{*}$ is a $C^{*}$-algebra, Theorem 2.4 is contained in [11, Theorem 9.1, Theorem 9.2, Theorem 9.3] and [26, Satz 3.6, Korollar 3.7].

For a moment let us turn to the commutative situation:

Corollary 2.6. Suppose $\left(R, R^{+}\right)$is a quasi-(0)-complete ([1, page 1164]) Banach space with a closed generating cone $R^{+}$and continuous involution, which has the Riesz interpolation (decomposition) property. Then there is one and only one way to define a matrix order on $R$ with closed cones in $M_{n}(R)$ such that $R_{1}^{+}=R^{+} . R_{n}^{+}$is given by

$$
R_{n}^{+}=\overline{\operatorname{co}}\left\{R^{+} \otimes M_{n}^{+}\right\}
$$




$$
=\left\{\sum_{\kappa=1}^{k} x_{\kappa} \otimes \alpha_{\kappa}, x_{\kappa} \in R^{+}, \alpha_{\kappa} \in M_{n}^{+}, k \in \mathrm{N}\right\}^{-} .
$$

$\left(R, R_{n}^{+}, n \in \mathrm{N}\right)$ has the approximative factorisation property.

Proof. We can assume without loss of generality that $\left(R, R^{+}\right)$is regular ordered in the sense of [25, Definition 2.2]. Now the uniqueness of the matrix order follows from [25, Theorem 3.1]. The second statement is a consequence of [27, Proposition 2.1] and Theorem 2.4.

One knows, that Banach lattices have the Riesz interpolation property Principle ideals of Banach lattices are AM-spaces and these are isometrically order isomorphic to the spaces $C(K), K$ a compact Hausdorf space by the representation Theorem of $M$. and S. Krein and S. Kakutani [19]. The following Theorem can be seen as a matricial analogue to this:

Theorem 2.7. Suppose $R$ and $R_{*}$ as in Theorem 2.4 and one of the conditions (I)-(VI) satisfied. If $\xi \in R_{n}^{+}$, then

$$
\left(V=\operatorname{span}_{\mathrm{C}} F_{\xi}, V_{m}^{+}=V_{m} \cap R_{n}^{+} \cdot m, m \in \mathrm{N}\right)
$$

is completely isomorphic to an injective $\mathrm{W}^{*}$-algebra $\mathscr{M}_{\xi}$. The isomorphism is $\sigma\left(\mathscr{M}_{\xi}, \mathscr{M}_{\xi *}\right)-\sigma\left(V, R_{*}\right)$ continuous.

Proof. Suppose first $n=1$. [6, Theorem 4.4] shows that $\left(V, V_{m}^{+}, m \in N\right)$ is completely order isomorphic to an operator system. $\left(V, V_{m}^{+}\right)$is injective since $R$ is an Arveson space. Hence $\left(V, V_{m}^{+}\right)$is completely isomorphic to an injective $C^{*}$-algebra by [6, Theorem 3.1]. Thus we have a new norm on $V$ :

$$
\|v\|_{\infty}=\inf \left\{t \in \mathrm{R}^{+}:\left[\begin{array}{cc}
t \xi & v \\
v^{*} & t \xi
\end{array}\right] \geqq 0\right\}
$$

and the unit ball $V_{B}$ of $\left(V,\|\cdot\|_{\infty}\right)$ is $\sigma\left(V, R_{*}\right)$ closed. If $0 \leqq v \leqq \xi$, then $\|v\|_{R} \leqq \lambda\|\xi\|_{R}$, since $R^{+}$is $\lambda$-normal $([17$, Theorem 3.2 and the remarks following Definition 2.2]). Hence $V_{B}$ is $\sigma\left(V, R_{*}\right)$ compact. If $v_{n}=v_{n}^{*} \rightarrow 0$ with respect to $\|\cdot\|_{\infty}$, then

$$
-\left\|v_{n}\right\|_{\infty} \xi \leqq v_{n} \leqq\left\|v_{n}\right\|_{\infty} \xi
$$

implies $v_{n} \rightarrow 0$ with respect to $\sigma\left(V, R_{*}\right)$. Now we apply Mackey's Theorem in order to show that the closure of $R_{*}$ in $V^{*}$ is a predual of $V$.

If $n>1$, then there exists $\gamma \in R^{+}$such that $\xi \leqq \gamma \otimes 1_{n}$. Hence $V$ can be considered as a subspace of the injective $\mathrm{W}^{*}$-algebra $\mathscr{M}_{\gamma} \otimes M_{n}$. This completes the proof. 


\section{Characterization of matrix ordered standard forms of injective $\mathrm{W}^{*}$-algebras.}

Theorem 3.1. Suppose $\left(\mathscr{M}, \mathscr{H}, \mathscr{H}_{n}^{+}, n \in \mathrm{N}\right)$ is the matrix ordered standard form of a $\mathrm{W}^{*}$-algebra $\mathscr{M}$ is injective (an Arveson space) if and only if $\mathscr{H}$ is an Arveson space.

Proof. (a) Suppose first that $\mathscr{M}$ is $\sigma$-finite. If $\varphi: N_{2 n} \rightarrow \mathscr{H}$ is a completely positive, linear map, then there exists a cyclic and separating vector $\xi \in \mathscr{H}^{+}$ such that $\varphi\left(1_{2 n}\right) \leqq \xi$. Lemma 1.4 implies that $\varphi$ can be considered as a completely positive map $\varphi: N_{2 n} \rightarrow \mathscr{M}$. If $\mathscr{M}$ is injective, then $\varphi$ has a completely positive extension to $M_{2 n}$. Hence $\mathscr{H}$ is an Arveson space by Theorem 2.4. The proof of the converse implication uses the same argument.

(b) Suppose $\mathscr{M}$ is arbitrary. Fix an increasing net $\left(p_{v}\right), v \in I$ of $\sigma$-finite projections converging to 1 and define $q_{v}=p_{v} J p_{v} J$. Then $\left(q_{v} \mathscr{M} q_{v}, q_{v} \mathscr{H}\left(q_{v} \otimes 1_{n}\right) \mathscr{H}_{n}^{+}, n \in \mathrm{N}\right)$ is a matrix ordered standard form of

and $\xi \in \mathscr{H}_{2 n}^{J}$ is $\sigma_{n, n}$-positive. Then $\left(q_{v} \otimes 1_{2 n}\right) \xi$ is $\sigma_{n, n}$-positive. By Theorem 2.4 we have $\eta_{v} \in q_{v} \mathscr{H}^{J}$ such that

$$
\eta_{v} \otimes \sigma_{n, n} \leqq\left(q_{v} \otimes 1_{2 n}\right) \xi \text {. }
$$

In particular $-q_{v} \xi_{2 n, 2 n} \leqq \eta_{v} \leqq q_{v} \xi_{1,1}$ implies without loss of generality $\eta_{v} \rightarrow \eta$ (weakly) and $\eta \otimes \sigma_{n, n} \leqq \xi$. Hence we have by Theorem 2.4 and (a):

$\mathscr{H}$ is an Arveson space

$\Leftrightarrow$ every $q_{v} \mathscr{H}$ is an Arveson space

$\Leftrightarrow$ every $q_{v} \mathscr{M} q_{v}$ is injective

$\Leftrightarrow \mathscr{M}$ is injective.

The last equivalence is well known and can be obtained in the same way as the first.

TheOREM 3.2. Suppose $\left(\mathscr{H}, \mathscr{H}_{n}^{+}, n \in \mathrm{N}\right)$ is a matrix ordered Hilbert space with selfdual cones $\mathscr{H}_{n}^{+} \subset \mathscr{H}_{n}$. Let $\mathscr{M}$ be the matrix multiplier algebra of $\left(\mathscr{H}, \mathscr{H}_{n}^{+}\right)$. If $\mathscr{H}$ satisfies one of the conditions (I)-(VI) of Theorem 2.4 , then $\mathscr{M}$ is injective and $\left(\mathscr{H}, \mathscr{H}, \mathscr{H}_{n}^{+}, n \in \mathrm{N}\right)$ is a matrix ordered standard form.

Proof. We apply Theorem 1.3, Theorem 2.4, Theorem 2.7 and Theorem 3.1.

Acknowledgement. I would like to express my gratitude to Prof. Dr. Gerd Wittstock, who brought the problem to my attention and with whom 
I had many fruitful conversations about the topic. I also thank the referee for several useful suggestions.

\section{REFERENCES}

1. Tsuyoshi Ando, On fundamental properties of a Banach space with a cone, Pacific J. Math. 12 (1962), 1163-1169.

2. Huzihiro Araki, Some properties of modular conjugation operator of von Neumann algebras and a non-commutative Radon-Nikodym Theorem with a chain rule, Pacific J. Math. 50 (1974), 309-354.

3. William B. Arveson, Subalgebras of C*-algebras I, Acta Math. 123 (1969), 141-224.

4. William G. Bade, The Banach space $C(S)$, Lecture Notes Series No. 26, Aarhus University, 1971.

5. Man-Duen Choi, Completely positive linear maps on complex matrices, Linear Algebra Appl. 10 (1975), 285-290.

6. Man-Duen Choi and Edward G. Effros, Injectivity and operator spaces, J. Funct. Anal. 24 (1977), 156-209.

7. Man-Duen Choi and Edward G. Effros, Nuclear $\mathrm{C}^{*}$-algebras and injectivity: the general case, Indiana Univ. Math. J. 26 (1977), 443-446.

8. Alain Connes, Caractérisation des éspaces vectoriels ordonnés sous-jacents aux algèbres de von Neumann, Ann. Inst. Fourier (Grenoble) 24 (1974), 121-155.

9. Alain Connes, Classification of injective factors, Ann. of Math. 104 (1976), 73-116.

10. Alain Connes, von-Neumann algebras (Proc. International Congr. Math., Helsinki, 1978), ed. O. Lehto, Vol. 1, pp. 97-109, Helsinki, 1980.

11. Edward G. Effros, Aspects of non-commutative order, in $\mathrm{C}^{*}$-algebras and applications to physics (Proc. 2nd Japan-USA Seminar, Los Angeles, 1977), eds. R. V. Kadison and H. Araki, (Lecture Notes in Math. 650), pp. 1-40. Springer-Verlag, Berlin - Heidelberg New York, 1978.

12. Edward G. Effros and Christopher Lance, Tensor products of operator algebras, Adv. in Math. 25 (1977), 1-34.

13. Uffe Haagerup, The standard form of von Neumann algebras, Math. Scand. 37 (1975), 271-283.

14. Morisake Hasumi, The extension property of complex Banach spaces, Tohoku Math. J. 10 (1958), 135-142.

15. Bruno Jochum, Cônes autopolaires et algèbres de Jordan (Lecture Notes in Math. 1049), Springer-Verlag, Berlin - Heidelberg - New York, 1984.

16. H. Elton Lacey, The isometric theory of classical Banach spaces (Grundlehren Math. Wiss. 208), Springer-Verlag, Berlin - Heidelberg - New York, 1974.

17. Jürgen Mangold and Rainer J. Nagel, Duality of cones in locally convex spaces, in Foundations of quantum mechanics and ordered linear spaces (Advanced Study Inst., Marburg, 1973), eds. A. Hartkämper and H. Neumann, (Lecture Notes in Physics 29), pp. 11-29. Springer-Verlag, Berlin - Heidelberg - New York, 1974.

18. Helmut H. Schaefer, Topological vector spaces, Macmillan Series in Advanced Mathematics and Theoretical Physics, The Macmillan Company, New York, 1966.

19. Helmut H. Schaefer, Banach lattices and positive operators (Grundlehren Math. Wiss. 215), Springer-Verlag, Berlin - Heidelberg - New York, 1974.

20. Lothar M. Schmitt, Characterization of, $L^{2}(\mathscr{M}), \mathscr{M}_{*}$ and $\mathscr{M}_{* *}$ for injective $\mathrm{W}^{*}$-algebras $\mathscr{M}$, preprint, Universität des Saarlandes, 1983. 
21. Lothar M. Schmitt and Gerd Wittstock, Characterization of matrix ordered standard forms of $\mathrm{W}^{*}$-algebras, Math. Scand. 51 (1982), 241-260.

22. Serban Strătilà and Lásló Zsidó, Lectures on von Neumann algebras, Abacus Press, Tunbridge Wells, England, 1979.

23. Masamichi Takesaki, Tomita's theory of modular Hilbert algebras and its applications (Lecture Notes in Math. 128), Springer-Verlag, Berlin - Heidelberg - New York, 1970.

24. Gerd Wittstock, Eine Bemerkung über Tensorprodukte von Banachverbänden, Arch. Math. (Basel) 25 (1974), 627-634.

25. Gerd Wittstock, Ordered normed tensor products, in Foundations of quantum mechanics and ordered linear spaces (Advanced Study Inst., Marburg, 1973), eds. A. Hartkämper and H. Neumann, (Lecture Notes in Physics 29), pp. 67-84. Springer-Verlag, Berlin Heidelberg - New York, 1974.

26. Gerd Wittstock, Ein operatorwertiger Hahn-Banach Satz, J. Funct. Anal. 40 (1981), 127150.

27. Gerd Wittstock, On matrix order and convexity, in Surveys and recent results, III, (Proc. 3rd Conf., Paderborn, 1983), eds. K.-D. Bierstedt and B. Fuchssteiner, Elsevier Science Publishers B. V. (North Holland), 1984.

\author{
UNIVERSTTÃT DES SAARLANDES \\ FACHBEREICH MATHEMATIK \\ D.6600 SAARBROCKEN \\ W. GERMANY
}

